\title{
Variability of displacements and stresses at random variable state
}

\author{
Hyuk Chun Noh ${ }^{\dagger}$ \\ Department of Civil and Environmental Engineering, Sejong University, Seoul, Korea \\ Phill Seung Lee \\ Department of Ocean Systems Engineering, Korea Advanced Institute of Science and Technology, \\ Daejeon, Korea \\ Chang Koon Choi \\ Department of Civil and Environmental Engineering, Korea Advanced Institute of Science and Technology, \\ Daejeon, Korea \\ (Received October 11, 2007, Accepted February 10, 2009)
}

\section{Introduction}

The statistical methodology, such as the Monte Carlo simulation (MCS), and expansion-based methodologies, including Karhunen-Loeve expansion, the polynomial chaos expansion (Ghanem and Spanos 1990), and the weighted integral method (Choi and Noh 1996, Noh 2006) provide generally acceptable approximation on the various stochastic problems. Despite the variety of analysis schemes, however, it is still difficult to obtain exact mathematical solutions of the statistical response of systems, even though a few is available in the literature such as Elishakoff and Ren (1995). The difficulties in the MCS lie on the fact that the numerically generated random samples, which are approximations in essence, should be used. In case of the expansion-based schemes the expansion order has to be truncated for computational availability, deteriorating the accuracy of the results. In particular, the discrepancies between numerical and theoretical results increase, if we deal with the higher order random parameters.

Here, we have concern on the response variability of structural systems in a special circumstance called random variable state, in which the correlation length of the stochastic field is infinite. Firstly, we derive exact expressions for the response variability of systems with Gaussian random elastic modulus in the random variable state. Then the results are compared with those evaluated

$\uparrow$ Assistant Professor, Ph.D., Corresponding author, E-mail: cpebach@sejong.ac.kr

+ Associate Professor, Ph.D., E-mail: phillseung@kaist.edu

t† Professor Emeritus, E-mail: cck@kaist.ac.kr 
using probability density function (p.d.f), which is possible for the special case of random variable state. Besides to the demonstration on the adequacy of the proposed schemes, some remarks concerning the limitations of Gaussian assumption on the material properties are addressed.

\section{Evaluation of response variability: use of p.d.f}

For the random variable state, any individual realization corresponds to a specific point on the abscissa of a given p.d.f, and the ordinate is the probability of occurrence of that realization. This means that for any specific realization $r_{i}$ we know exactly the probability of occurrence $p_{i}(r)$. For a pre-assigned criterion $\varepsilon$, the random variable $R$ has its realization in the range $\left[r_{L}, r_{R}\right]$, where $\operatorname{prob}(r)<\varepsilon \forall r \notin\left[r_{L}, r_{R}\right]$. The criterion $\varepsilon$ is determined based on the precision of the analysis. Dividing the range $\left[r_{L}, r_{R}\right]$ into $N_{s}$ subdivisions, we can have $N_{s}$ number of random samples $r_{i}$ of which the probability can be written as $p_{i}(r)=p\left(r_{i}\right) d r$, where $d r$ is $\left(r_{R}-r_{L}\right) / N_{s}$.

We can evaluate the $i$-th response $u_{i}$ based on the random material property $E_{i}=\bar{E}\left(1+r_{i}\right)$. Here, of course, the probability of occurrence of $u_{i}, p_{i}(u)$, is equal to that of the random realization $r_{i}$, i.e., $p_{i}(u)=p_{i}(r)$, which enable us to establish the p.d.f of the response $u$, i.e., $p\left(u_{i}\right)=p\left(r_{i}\right) d r / d u$. Once we have p.d.f of the response, it is straight forward to obtain the first and second moment of the response. The discrete version of the mean and variance, $\mu_{u}$ and $\sigma_{u}^{2}$ respectively, are

$$
\mu_{u}=\sum_{i=1}^{N_{s}} p_{i}(u) \Delta u ; \quad \sigma_{u}^{2}=\sum_{i=1}^{N_{s}}\left(u_{i}-\mu_{u}\right)^{2} p\left(u_{i}\right) \Delta u
$$

\section{Evaluation of response variability: exact expressions}

As an alternative for evaluations of statistical responses for the random variable state, we derive exact expressions for the mean and variance of the response. Since the stochastic field becomes a constant field as $f(\mathbf{x})=\alpha$ for $\forall \mathbf{x} \in \Omega_{\text {str }}$, where $\Omega_{\text {str }}$ stands for entire domain of the structure, the global stiffness of the system becomes $\mathbf{K}=(1+\alpha) \mathbf{K}_{o}$, and therefore the displacement becomes

$$
\mathbf{U}=\mathbf{K}^{-1} \mathbf{F}=\frac{1}{1+\alpha} \mathbf{K}_{o}^{-1} \mathbf{F}=\sum_{k=0}^{\infty}(-1)^{k} \alpha^{k} \mathbf{U}_{o}, \quad \mathbf{U}_{o}=\mathbf{K}_{o}^{-1} \mathbf{F}
$$

Applying the mean operator to Eq. (2), the mean of the response is evaluated to be $\overline{\mathbf{U}}=(1+\mu) \mathbf{U}_{o}$, where the symbol $\mu$ denotes the expectation on the sum of the random variable $\alpha$ in even power. Employing the recursive formula for the $n$-th joint moment (Lin 1967), each random variable in even power can be transformed into a series of repeated auto-correlation functions resulting in Eq. (3), where stands for the coefficient of variation (COV) of the stochastic field

$$
\mu=E\left[\sum_{k=1}^{\infty} \alpha^{2 k}\right]=\sum_{k=1}^{\infty} \frac{(2 k) !}{k ! 2^{k}} \sigma_{f}^{2 k}
$$

Using the mean displacement, the deviation of the displacement with respect to the mean is given as $\delta \mathbf{U}=\left(-\alpha+\alpha^{2}-\alpha^{3}+\ldots-\mu\right) \mathbf{U}_{o}$, therefore the covariance of the displacement can be established in a straightforward way as follows

$$
\operatorname{Cov}[\mathbf{U}, \mathbf{U}]=\left\{5 \mu-\mu^{2}-\left(4 \sigma_{f}^{2}+6 \sigma_{f}^{4}\right)\right\} \mathbf{U}_{o}^{2}
$$


In the case of stresses, the mean stress is derived to be the same as the deterministic stress, and therefore, the covariance of the stresses is vanished, i.e., $\bar{\sigma}=\sigma_{o}$ and $\operatorname{Cov}[\sigma, \sigma]=0.0$.

\section{Numerical verifications and remarks}

Firstly, we have investigated into the acceptability of the Gaussian distribution for the random elastic modulus. As contrary to the expectation, the mean displacement $\overline{\mathbf{U}}=(1+\mu) \mathbf{U}_{o}$ does not converge as the summation index $k$ in Eq. (3) increases, but diverges after a certain $k_{d}$, which depends on the COV of the stochastic field. The larger the COV of the stochastic field, the smaller the $k_{d}$.

Fig. 1(a) shows the diverging phenomenon of the mean displacement in terms of the rate of change of the mean displacement when the COV of the stochastic field is 0.225 . This phenomenon appears due to the effect of negative tail of the p.d.f, which has relatively higher probability when the COV is high and cause a non-physical elastic modulus, zero or negative. In Fig. 1(b), the index $k_{d}$, the summation index just before the mean displacement diverges, is given in terms of the COV of the stochastic field. This result tells us that even though we might obtain a certain result, that result is not a converged one and even could not reach convergence.

Even though the Gaussian assumption on the random elastic modulus is not acceptable as already addressed, some meaningful results can be obtained within tolerable errors. In order to investigate into the response variability, we take a $20 \times 20$ square plate as an example. For material constants we take: the elastic modulus $E=10920.0$, the Poisson's ratio $v=0.3$. The distributed unit load is applied on the upper face of the plate. Considering the symmetry in the structure as well as the applied load, a quarter model is employed.

Fig. 2 displays the p.d.f and cumulative distribution function (c.d.f) of the random variable and the response $u$. The p.d.f of the response is normalized with respect to the mean response. As shown in Fig. 2, the response is not Gaussian and the deviation from the Gaussian p.d.f becomes pronounce for larger COV of the stochastic field.
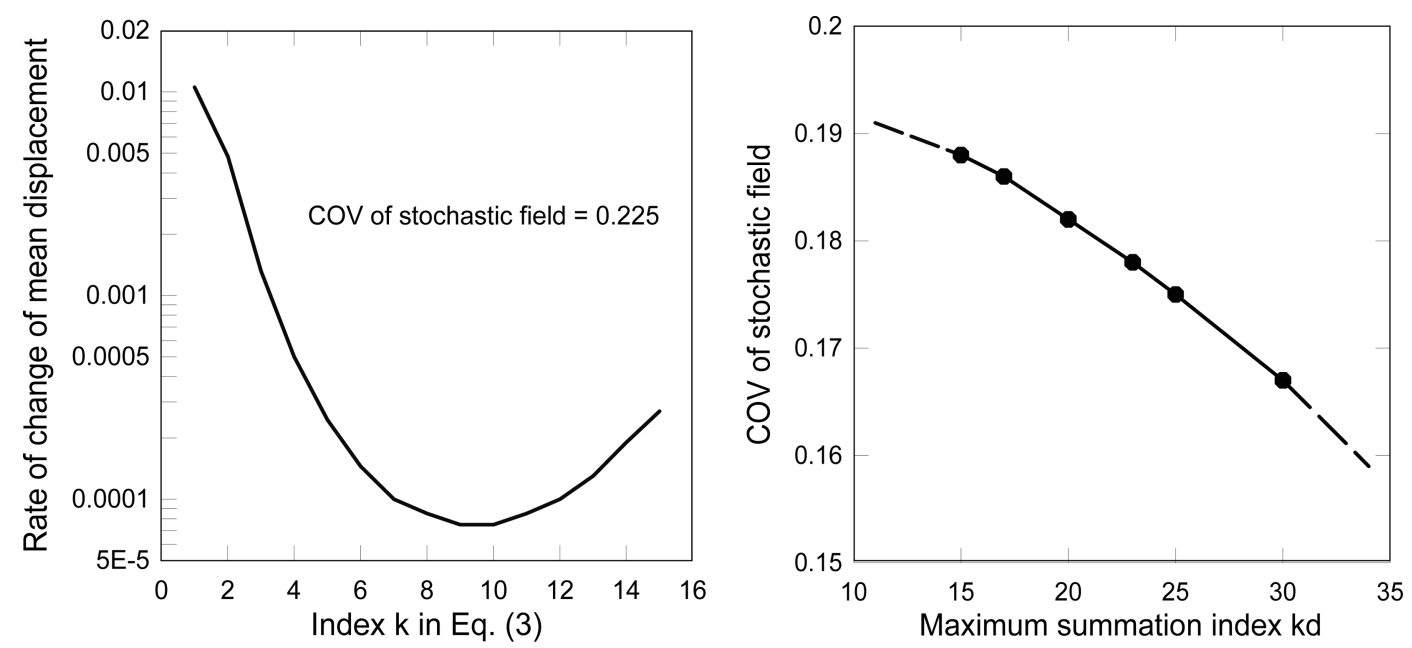

Fig. 1 (a) Divergence of the mean displacement and (b) COV of the stochastic field and index $k_{d}$ 

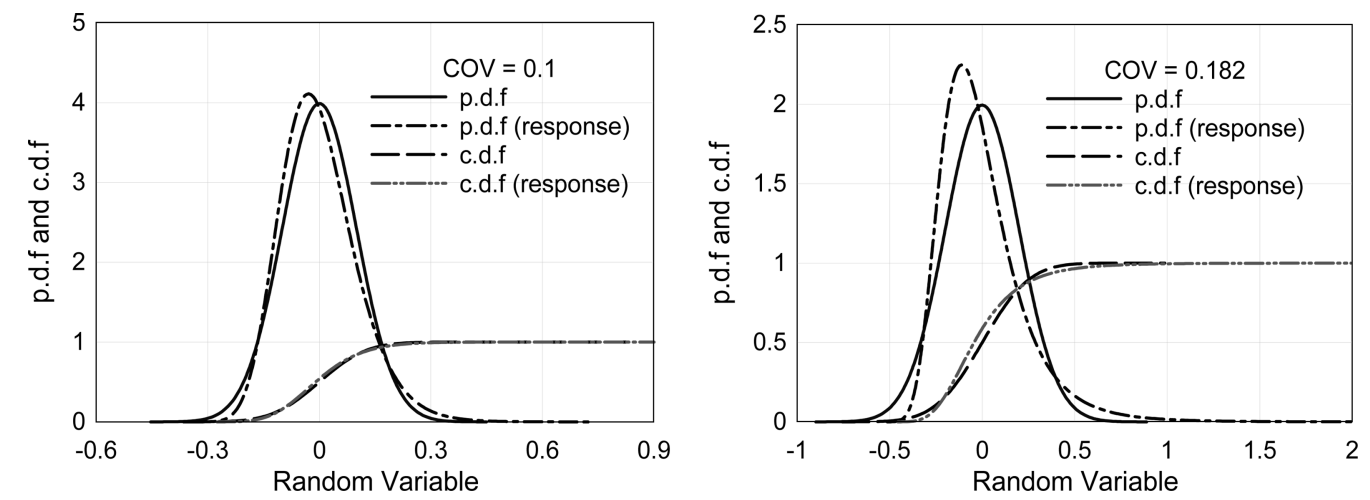

Fig. 2 p.d.f and c.d.f for random variable and response: (a) Coefficient of variation $=0.10$, (b) Coefficient of variation $=0.182$

Table 1 Mean and COV of response (vertical translation at the center of the plate)

\begin{tabular}{|c|c|c|c|c|c|c|}
\hline $\begin{array}{l}\text { COV of random } \\
\text { elastic modulus }\end{array}$ & Schemes & $\begin{array}{c}\text { Mean } \\
\text { displacement }\end{array}$ & $1+\mu$ & $\begin{array}{l}\text { Response } \\
\text { COV }^{(b)}\end{array}$ & Ratio (b)/(a) & Ratio $(d) /(c)$ \\
\hline \multirow{2}{*}{0.100} & Using p.d.f & $6.6518 \times 10^{-1}$ & 1.010316 & $0.103228^{(\mathrm{c})}$ & 1.032 & \multirow{2}{*}{0.9999} \\
\hline & Exact expressions & $6.6518 \times 10^{-1}$ & 1.010316 & $0.103215^{(\mathrm{d})}$ & 1.032 & \\
\hline \multirow{2}{*}{0.182} & Using p.d.f & $6.8285 \times 10^{-1}$ & 1.037154 & $0.207374^{(\mathrm{c})}$ & 1.139 & \multirow{2}{*}{0.9895} \\
\hline & Exact expressions & $6.8285 \times 10^{-1}$ & 1.037150 & $0.205194^{(\mathrm{d})}$ & 1.127 & \\
\hline
\end{tabular}

Table 1 shows the results obtained using both the p.d.f and exact expressions. As shown, the two schemes are in good agreement, in addition, it is also noticed that the difference between the response $\mathrm{COV}$ and $\mathrm{COV}$ of the stochastic field is increases as the randomness of the stochastic field increases.

From the comparison of the results between the proposed and those in the literature (Noh 2004, 2006), where elastic modulus and Poissons ratio are taken into account simultaneously as random parameters, we found that the proposed schemes provide upper bounds.

\section{References}

Choi, C.K. and Noh, H.C. (1996), "Stochastic finite element analysis of plate structures by weighted integral method", Struct. Eng. Mech., 4(6), 703-715.

Deodatis, G., Graham-Brady L. and Micaletti, R. (2003), "A hierarchy of upper bounds on the response of stochastic systems with large variation of their properties: Random variable case", Probabilist. Eng. Mech., 18, 349-363.

Elishakoff, I. and Ren, Y.J. (1995), "Some exact solutions for the bending of beams with spatially stochastic stiffness", Int. J. Solids Struct., 32(6), 2315-2327.

Ghanem, R.G. and Spanos, P.D. (1990), "Polynomial chaos in stochastic finite elements", J. Appl. Mech., 57, $197-202$.

Lin, Y.K. (1967), Probabilistic Theory of Structural Dynamics, McGraw-Hill book Company.

Noh, H.C. (2004), "A formulation for stochastic finite element analysis of plate structures with uncertain Poisson's ratio", Comput. Meth. Appl. Mech. Eng., 193(45-47), 4857-4873.

Noh, H.C. (2006), "Effect of multiple uncertain material properties on statistical behavior of in-plane and plate structures", Comput. Meth. Appl. Mech. Eng., 195(19-22), 2697-2718. 